\title{
ON MATRIX POLYNOMIALS ASSOCIATED WITH HERMITE MATRIX POLYNOMIALS
}

\author{
M. A. PATHAN, MAGED G. BIN-SAAD AND FADHL AL-SARHI
}

\begin{abstract}
In this paper, an extension of the Hermite matrix polynomials is introduced. Some relevant matrix functions appear in terms of the two-index and two-variable and p-index and p-variable Hermite matrix polynomials. Furthermore, in order to give qualitative properties of this family of matrix polynomials, the Legendre and Chebyshev matrix polynomials of sveral variables are introduced.
\end{abstract}

\section{Introduction}

An extension to the matrix framework of the classical families of Laguerre, Hermite and Gegenbauer polynomials have been introduced in $([4,5,6])$. In [3], the Laguerre and Hermite matrix polynomials are presented as examples of right orthogonal matrix polynomial sequences for appropriate right matrix moment functional of integral type.

Jodar and Company [4] introduced the class of Hermite matrix polynomials $H_{n}(x, A)$ defined by

$$
\exp \left(x t \sqrt{(2 A)}-t^{2} I\right)=\sum_{n=0}^{\infty} H_{n}(x, A) \frac{t^{n}}{n !},
$$

where

$$
H_{n}(x, A)=n ! \sum_{k=0}^{\left[\frac{n}{2}\right]} \frac{(-1)^{k}(\sqrt{2 A})^{n-2 k}}{k !(n-k) !} x^{n-2 k}, n \geq 0,
$$

where $A$ is a positive stable matrix in the complex space $C^{N \times N}$ of all square matrices of common order $N$, which appear as a finite series solutions of second order matrix differential equations $y^{\prime \prime}-x A y^{\prime}+n A y=0$,for a matrix $A$ in $C^{N \times N}$ whose eigen values are all in the right open half-plane. In [1], Batahan presented a study of the two-variable Hermite matrix polynomials defined by

$$
\exp \left(x t \sqrt{\left.(2 A)-y t^{2} I\right)}=\sum_{n=0}^{\infty} H_{n}(x, y, A) \frac{t^{n}}{n !},\right.
$$

Received August 10, 2014, accepted October 7, 2014. 2010 Mathematics Subject Classification. 33C25, 33C45, 33E20, 15A60.

Key words and phrases. Hypergeometric function, Hermite matrix polynomials, generating function, Legendre and Chebyshev matrix polynomials.

Corresponding author: M. A. Pathan. 
where

$$
H_{n}(x, y, A)=n ! \sum_{k=0}^{\left[\frac{n}{2}\right]} \frac{(-1)^{k}(\sqrt{2 A})^{n-2 k}}{k !(n-k) !} x^{n-2 k} y^{k}, n \geq 0 .
$$

Now, we recall that if $A(k, n)$ and $B(k, n)$ are matrices in $C^{N \times N}$ for $n \geq 0$ and $k \geq 0$,then it follows that [8]:

$$
\begin{aligned}
& \sum_{n=0}^{\infty} \sum_{k=0}^{n} A(k, n)=\sum_{n=0}^{\infty} \sum_{k=0}^{\infty} A(k, n+k), \\
& \sum_{n=0}^{\infty} \sum_{k=0}^{\infty} B(k, n)=\sum_{n=0}^{\infty} \sum_{k=0}^{\left[\frac{n}{2}\right]} B(k, n-2 k), \\
& \sum_{n=0}^{\infty} \sum_{k=0}^{2 n} A(k, n)=\sum_{n=0}^{\infty} \sum_{k=0}^{n} A(2 k, n) .
\end{aligned}
$$

For $m$ is a positive integer, we can write

$$
\sum_{n=0}^{\infty} \sum_{k=0}^{n} A(k, n)=\sum_{n=0}^{\infty} \sum_{k=0}^{[n / m]} A(k, n-(m-1) k) .
$$

In the course of an attempt to unify several results in the theory of matrix polynomials of more than one variable, we define Hermite matrix polynomials of several index and several variables. The importance of this new class of matrix polynomials have been recognized both in purely mathematical and applied frame works. The structure of this paper is the following: In section 2, we introduce and study the two-index and two-variable Hermite matrix polynomials $H_{n, m}(x, y ; A)$. In sections 3 and 4,we study a number of properties of the two-index two-variable Hermite matrix polynomials involving recurrence relations and Rodrigue's formula respectively. In section 5 , we introduce and study the multiindices and multivariable Hermite matrix polynomials $H_{n_{1}, n_{2}, \ldots, n_{p}}\left(x_{1}, x_{2}, \ldots, x_{p} ; A\right)$ and obtain a number of properties of the $\mathrm{p}$-index and $\mathrm{p}$-variable Hermite matrix polynomials involving recurrence relations and Rodrigue's formula. Finally, in section 6 definitions of p-index and p-variable Legendre and Chebyshev matrix polynomials are introduced as a new families of polynomials.

\section{Two-index and two-variable Hermite matrix polynomials}

Let $A$ be a positive stable matrix in $C^{N \times N}$.We define two-index and two-variable Hermite matrix polynomials by means of the following generating function:

$$
f(x, y, t, h)=\exp \left(x(t+h) \sqrt{\left.(2 A)-y(t+h)^{2} I\right)}=\sum_{n, m=0}^{\infty} H_{n, m}(x, y ; A) \frac{t^{n} h^{m}}{n ! m !} .\right.
$$

Making use of the matrix exponential series

$$
e^{x}=\sum_{n=0}^{\infty} \frac{x^{n}}{n !}
$$


and the binomial expansion

$$
(a+x)^{n}=\sum_{r=0}^{n}\left(\begin{array}{l}
n \\
r
\end{array}\right) a^{n-r} x^{r}
$$

we obtain

$$
f(x, y, t, h)=\sum_{n=0}^{\infty} \sum_{m=0}^{n} \sum_{r=0}^{\infty} \sum_{s=0}^{2 r} \frac{(-1)^{r}(2 r) !(x \sqrt{(2 A)})^{n} y^{r}}{m ! r ! s !(n-m) !(2 r-s) !} t^{n-m+2 r-s} h^{m+s},
$$

which in view of the equations (1.5), (1.6) and (1.8) gives us

$$
\begin{aligned}
f(x, y, t, h) & =\sum_{n=0}^{\infty} \sum_{m=0}^{n} \sum_{r=0}^{\infty} \sum_{s=0}^{r} \frac{(-1)^{r}(2 r) !(x \sqrt{(2 A)})^{n} y^{r}}{m ! r !(2 s) !(n-m) !(2 r-2 s) !} t^{n-m+2 r-2 s} h^{m+2 s}, \\
& =\sum_{n=0}^{\infty} \sum_{m=0}^{\infty} \sum_{r=0}^{\infty} \sum_{s=0}^{\infty} \frac{(-1)^{r+s}(2 r+2 s) !(x \sqrt{(2 A)})^{n+m} y^{r+s}}{(r+s) !(2 r) !(2 s) ! n ! m !} t^{n+2 r} h^{m+2 s}, \\
& =\sum_{n, m=0}^{\infty} \sum_{r=0}^{\left[\frac{n}{2}\right]\left[\frac{m}{2}\right]} \sum_{s=0}(-1)^{r+s} \frac{(2 r+2 s) ! y^{r+s}(x \sqrt{2 A})^{n+m-2 r-2 s} t^{n} h^{m}}{(r+s) !(n-2 r) !(m-2 s) !(2 r) !(2 s) !} .
\end{aligned}
$$

Thus, from (2.1) and (2.3), we obtain the following explicit representation for the two-index and two-variable Hermite matrix polynomials

$$
H_{n, m}(x, y ; A)=n ! m ! \sum_{r=0}^{\left[\frac{n}{2}\right]} \sum_{s=0}^{\left[\frac{m}{2}\right]}(-1)^{r+s} \frac{(2 r+2 s) ! y^{r+s}(x \sqrt{2 A})^{n+m-2 r-2 s}}{(n-2 r) !(m-2 s) !(2 r) !(2 s) !(r+s) !} .
$$

For $m=0$, (2.4) reduces to (1.4). Further, it is of interest to point out that the series representation in (2.4), in particlar, yields the following relationships:

$$
\begin{gathered}
H_{0,0}(x, y ; A)=I, \quad H_{n, 0}(x, y ; A)=H_{n}(x, y, A), \quad H_{1,1}(x, y ; A)=(x \sqrt{2 A})^{2}, \\
H_{n, m}(x, 0 ; A)=(x \sqrt{2 A})^{n+m}, \quad H_{n, m}(x, 1 ; A)=H_{n, m}(x ; A)
\end{gathered}
$$

Also, we can write

and

$$
H_{n, m}(x, y ; A)=y^{\frac{n+m}{2}} H_{n, m}\left(\frac{x}{\sqrt{y}} ; A\right)
$$

$$
H_{n, m}(-x, y ; A)=(-1)^{n+m} H_{n, m}(x, y ; A) .
$$

For $y=1$, it follows that

$$
\sum_{n, m=0}^{\infty} H_{n, m}(x ; A) \frac{t^{n} h^{m}}{n ! m !}=\exp \left(x(t+h) \sqrt{\left.(2 A)-(t+h)^{2} I\right),}\right.
$$

which when $x=0$ gives us

$$
\sum_{n, m=0}^{\infty} H_{n, m}(0 ; A) \frac{t^{n} h^{m}}{n ! m !}=\sum_{n, m-0}^{\infty} \frac{(-1)^{n+m}(2 n+2 m) !}{(n+m) !(2 n) !(2 m) !} t^{2 n} h^{2 m} .
$$


Therefore, we get

and

$$
H_{2 n, 2 m}(0 ; A)=(-1)^{n+m} 2^{2 n+2 m}\left(\frac{1}{2}\right)_{n+m} I,
$$

$$
H_{2 n+1,2 m+1}(0 ; A)=0 \text {. }
$$

Moreover, from (2.1), we have

$$
\sum_{n=0}^{\infty} \frac{(x \sqrt{2 A})^{n}}{n !}(t+h)^{n}=\sum_{r=0}^{\infty} \frac{y^{r}}{r !}(t+h)^{2 r} \times \sum_{n, m=0}^{\infty} \frac{H_{n, m}(x, y ; A)}{n ! m !} t^{n} h^{m},
$$

which can be written in the form

$$
\sum_{n, m=0}^{\infty} \frac{(x \sqrt{2 A})^{n+m}}{(n-m) ! m !} t^{n} h^{m}=\sum_{n, m, r, s=0}^{\infty} \frac{(2 r+2 s) ! y^{r+s} H_{n, m}(x, y ; A)}{n ! m !(r+s) !(2 s) !(2 r) !} t^{n+2 r} h^{m+2 s} .
$$

Next, applying (1.6), equation (2.7) gives us

$$
\sum_{n, m=0}^{\infty} \frac{(x \sqrt{2 A})^{n+m}}{n ! m !} t^{n} h^{m}=\sum_{n, m=0}^{\infty} \sum_{r=0}^{\left[\frac{n}{2}\right]} \sum_{s=0}^{\left[\frac{m}{2}\right]} \frac{(2 r+2 s) ! y^{r+s} H_{n-2 r, m-2 s}(x, y ; A)}{(n-2 r) !(m-2 s) !(r+s) !(2 s) !(2 r) !} t^{n} h^{m} .
$$

Now, on comparing the coefficient of $t^{n} h^{m}$, we obtain the following expansion formula:

Theorem 2.1. Let $A$ be a positive stable matrix in $C^{N \times N}$, then, we have

$$
(x \sqrt{2 A})^{n+m}=\sum_{r=0}^{\left[\frac{n}{2}\right]} \sum_{s=0}^{\left[\frac{m}{2}\right]}\left(\begin{array}{c}
n \\
2 r
\end{array}\right)\left(\begin{array}{l}
m \\
2 s
\end{array}\right)\left(\frac{1}{2}\right)_{r+s} 2^{2 r+2 s} y^{r+s} H_{n-2 r, m-2 s}(x, y ; A) .
$$

\section{Differential recurrence relations}

This section deals with some differential recurrence relations for the two-index two-variable Hermite matrix polynomials. First, we record the following theorem.

Theorem 3.1. The two-index two-variable Hermite matrix polynomials satisfies the following relations:

$$
\frac{\partial^{k}}{\partial x^{k}} H_{n, m}(x, y ; A)=(\sqrt{2 A})^{k} \sum_{r=0}^{k} \frac{n ! m ! k ! H_{n-(k-r), m-r}(x, y ; A)}{(n-(k-r)) !(m-r) !(k-r) ! r !}
$$

and

$$
\frac{\partial^{k}}{\partial y^{k}} H_{n, m}(x, y ; A)=(-1)^{k} \sum_{r=0}^{2 k} \frac{(2 k) ! n ! m ! H_{n-(2 k-r), m-r}(x, y ; A)}{(n-(2 k-r)) !(m-r) !(2 k-r) ! r !}
$$


Proof. Differentiating partially (2.1) with respect to " $x$ " , we get

$$
\sum_{n, m=0}^{\infty} \frac{\partial}{\partial x} H_{n, m}(x, y ; A) \frac{t^{n} h^{m}}{n ! m !}=(t+h) \sqrt{(2 A)} \exp \left(x(t+h) \sqrt{(2 A)}-y(t+h)^{2} I\right) .
$$

From (2.1) and (3.3), we have

$$
\sum_{n, m=0}^{\infty} \frac{\partial}{\partial x} H_{n, m}(x, y ; A) \frac{t^{n} h^{m}}{n ! m !}=\sqrt{(2 A)} \sum_{n, m=0}^{\infty} \sum_{r=0}^{1} \frac{H_{n-(1-r), m-r}(x, y ; A) t^{n} h^{m}}{r !(1-r) !(n-(1-r)) !(m-r) !} .
$$

On comparing the coefficient of $t^{n} h^{m}$ in (3.4), we obtain

$$
\frac{\partial}{\partial x} H_{n, m}(x, y ; A)=\sqrt{2 A} \sum_{r=0}^{1} \frac{n ! m !}{r !(1-r) !(n-(1-r)) !(m-r) !} H_{n-(1-r), m-r}(x, y ; A),
$$

Again, by differentiating (3.5) with respect to " $x$, we get

$$
\frac{\partial^{2}}{\partial x^{2}} H_{n, m}(x, y ; A)=2 A \sum_{r=0}^{2} \frac{2 n ! m ! H_{n-(2-r), m-r}(x, y ; A)}{r !(2-r) !(n-(2-r)) !(m-r) !},
$$

Iteration (3.5), for $0 \leq k \leq n$, implies (3.1). To prove (3.2), differentiate partially (2.1) with respect to " $y$ ", to get

$$
\sum_{n, m=0}^{\infty} \frac{\partial}{\partial y} H_{n, m}(x, y ; A) \frac{t^{n} h^{m}}{n ! m !}=(-1)(t+h)^{2} \exp \left(x(t+h) \sqrt{\left.(2 A)-y(t+h)^{2} I\right) .}\right.
$$

From (2.1) and (3.7), we have

$$
\sum_{n, m=0}^{\infty} \frac{\partial}{\partial y} H_{n, m}(x, y ; A) \frac{t^{n} h^{m}}{n ! m !}=(-1) \sum_{n, m=0}^{\infty} \sum_{r=0}^{2} \frac{2 H_{n-(2-r), m-r}(x, y ; A) t^{n} h^{m}}{r !(2-r) !(n-(2-r)) !(m-r) !} .
$$

On comparing the coefficient of $t^{n} h^{m}$ in (3.8), we obtain

$$
\frac{\partial}{\partial y} H_{n, m}(x, y ; A)=(-1) \sum_{r=0}^{2} \frac{2 n ! m ! H_{n-(2-r), m-r}(x, y ; A)}{r !(2-r) !(n-(2-r)) !(m-r) !},
$$

On iteration (3.9), for $0 \leq k \leq\left[\frac{n}{2}\right]$, we arrive at (3.2).

Corollary 3.1. The two-index two-variable Hermite matrix polynomials satisfies the following relations

$$
\frac{\partial^{2}}{\partial x^{2}} H_{n, m}(x, y ; A)+2 A \frac{\partial}{\partial y} H_{n, m}(x, y ; A)=0,
$$

in general

$$
\frac{\partial^{2 k}}{\partial x^{2 k}} H_{n, m}(x, y ; A)=(-1)^{k}(2 A)^{k} \frac{\partial^{k}}{\partial y^{k}} H_{n, m}(x, y ; A),
$$

Proof. On equating (3.6) and (3.9), we obtain (3.10), and equating (3.1) and (3.2), we get (3.11). 
Theorem 3.2. The two-index two-variable Hermite matrix polynomials satisfies the following relation:

$$
x \frac{\partial}{\partial x} H_{n, m}(x, y ; A)+2 y \frac{\partial}{\partial y} H_{n, m}(x, y ; A)-(n+m) H_{n, m}(x, y ; A)=0,
$$

Proof. Differentiating (2.1) with respect to $x, y, t$ and $h$, we find respectively

$$
\begin{aligned}
& \frac{\partial F}{\partial x}=(t+h) \sqrt{2 A} \exp \left[x(t+h) \sqrt{2 A}-y(t+h)^{2} I\right], \\
& \frac{\partial F}{\partial y}=-(t+h)^{2} \exp \left[x(t+h) \sqrt{2 A}-y(t+h)^{2} I\right], \\
& \frac{\partial F}{\partial t}=[x \sqrt{2 A}-2 y(t+h) I] \exp \left[x(t+h) \sqrt{2 A}-y(t+h)^{2} I\right], \\
& \frac{\partial F}{\partial h}=[x \sqrt{2 A}-2 y(t+h) I] \exp \left[x(t+h) \sqrt{2 A}-y(t+h)^{2} I\right] .
\end{aligned}
$$

From the above equations, we observe that:

$$
x \frac{\partial F}{\partial x}+2 y \frac{\partial F}{\partial y}-t \frac{\partial F}{\partial t}-h \frac{\partial F}{\partial h}=0,
$$

which again in view of (2.1) and identifying the coefficients of $t^{n} h^{m}$ on both sides gives (3.12). Therefore, the equation (3.12) is established and the proof of Theorem 3.2 is completed.

Corollary 3.2. The two-index two-variable Hermite matrix polynomials satisfies the following partial differential equation:

$$
\left[y \frac{\partial^{2}}{\partial x^{2}}-x A \frac{\partial}{\partial x}+(n+m) A\right] H_{n, m}(x, y ; A)=0, \quad n, m \geq 0 .
$$

Proof. From relations (3.5), (3.6)and (3.9), it is easily seen that

$$
\begin{aligned}
& \frac{\partial}{\partial x} H_{n, m}(x, y ; A)=\sqrt{2 A} n H_{n-1, m}(x, y ; A)+\sqrt{2 A} m H_{n, m-1}(x, y ; A), \\
& \frac{\partial^{2}}{\partial x^{2}} H_{n, m}(x, y ; A) \\
& \quad=2 A\left[n(n-1) H_{n-2, m}(x, y ; A)+2 n m H_{n-1, m-1}(x, y ; A)+m(m-1) H_{n, m-2}(x, y ; A)\right],
\end{aligned}
$$

and

$$
\begin{aligned}
& \frac{\partial}{\partial y} H_{n, m}(x, y ; A) \\
& \quad=-\left[n(n-1) H_{n-2, m}(x, y ; A)+2 n m H_{n-1, m-1}(x, y ; A)+m(m-1) H_{n, m-2}(x, y ; A)\right],
\end{aligned}
$$

respectively. Now, by starting from (3.12) and employing the above results we obtain (3.13). Therefore, the result is established. Now, we derive an operational representation for the Hermite matrix polynomials. 
Theorem 3.3. Let A be a matrix in $C^{N \times N}$. Then the two-index and two-variable Hermite matrix polynomials has the following representation:

$$
H_{n, m}(x, y ; A)=\exp \left(-y(2 A)^{-1} \frac{\partial^{2}}{\partial x^{2}}\right)(x \sqrt{2 A})^{n+m} .
$$

Proof. It is clear that

$$
\left[(\sqrt{2 A})^{-1} \frac{\partial}{\partial x}\right]^{2} \exp (x(t+h) \sqrt{2 A})=(t+h)^{2} \exp (x(t+h) \sqrt{2 A}) .
$$

Thus

$$
\exp \left[-y(\sqrt{2 A})^{-2} \frac{\partial^{2}}{\partial x^{2}}\right] \exp (x(t+h) \sqrt{2 A})=\sum_{n=0}^{\infty} \frac{(-1)^{n} y^{n}}{n !}(t+h)^{2 n} \exp (x(t+h) \sqrt{2 A}) .
$$

Also, we can write (4.3) in the form

$$
\exp \left[-y(\sqrt{2 A})^{-2} \frac{\partial^{2}}{\partial x^{2}}\right]^{2} \exp (x(t+h) \sqrt{2 A})=\exp \left(x(t+h) \sqrt{2 A}-y(t+h)^{2} I\right) .
$$

Therefore, by using (2.1), we obtain

$$
\exp \left[-y(\sqrt{2 A})^{-2} \frac{\partial^{2}}{\partial x^{2}}\right] \sum_{n, m=0}^{\infty} \frac{(x \sqrt{2 A})^{n+m}}{n ! m !} t^{n} h^{m}=\sum_{n, m=0}^{\infty} H_{n, m}(x, y ; A) \frac{t^{n} h^{m}}{n ! m !},
$$

which on comparing the coefficients of $t^{n} h^{m}$ yields (3.14).

\section{4. p-index and p-variable Hermite matrix polynomials}

Let $A$ be a positive stable matrix in $C^{N \times N}$. We define the p-index and p-variable Hermite matrix polynomials by means of the generating function

$$
\begin{aligned}
& \exp \left[x_{1}\left(t_{1}+\cdots+t_{p}\right) \sqrt{(2 A)}-\left(x_{2}+\cdots+x_{p}\right)\left(t_{1}+\cdots+t_{p}\right)^{2} I\right] \\
& =\sum_{n_{1}, \ldots, n_{p}=0}^{\infty} H_{n_{1}, \ldots, n_{p}}\left(x_{1}, \ldots, x_{p} ; A\right) \frac{t_{1}^{n_{1}}}{n_{1} !} \cdots \frac{t_{p}^{n_{p}}}{n_{p} !} .
\end{aligned}
$$

Proceeding on the same lines as in the proof of (2.4), the following explicit representation for the $\mathrm{p}$-index and p-variable Hermite matrix polynomials can be obtained

$$
\begin{aligned}
H_{n_{1}, \ldots, n_{p}}\left(x_{1}, \ldots, x_{p} ; A\right)= & n_{1} ! \cdots n_{p} ! \sum_{r_{1}=0}^{\left[\frac{n_{1}}{2}\right]} \cdots \sum_{r_{p}=0}^{\left[\frac{n_{p}}{2}\right]} \frac{(-1)^{r_{1}+\cdots+r_{p}}\left(2 r_{1}+\cdots+2 r_{p}\right) !}{\left(r_{1}+\cdots+r_{p}\right) !\left(n_{1}-2 r_{1}\right) ! \cdots\left(n_{p}-2 r_{p}\right) !} \\
& \frac{\left(x_{1} \sqrt{2 A}\right)^{n_{1}+\cdots+n_{p}-2 r_{1}-\cdots-2 r_{p}}\left(x_{2}+\cdots+x_{p}\right)^{r_{1}+\cdots+r_{p}}}{\left(2 r_{1}\right) ! \cdots\left(2 r_{p}\right) !}
\end{aligned}
$$


It is clear that

$$
\begin{aligned}
& H_{0, \ldots, 0}\left(x_{1}, \ldots, x_{p} ; A\right)=I, \quad H_{n, 0, \ldots, 0}\left(x_{1}, \ldots, x_{p} ; A\right)=H_{n}\left(x_{1}, \ldots, x_{p} ; A\right) \\
& H_{1, \ldots, 1}\left(x_{1}, \ldots, x_{p} ; A\right)=\left(x_{1} \sqrt{2 A}\right)^{n} \text { and } H_{n_{1}, \ldots, n_{p}}\left(x_{1}, 0, \ldots, 0 ; A\right)=\left(x_{1} \sqrt{2 A}\right)^{n_{1}+\cdots+n_{p}} .
\end{aligned}
$$

Also, we can write

$$
H_{n_{1}, \ldots, n_{p}}\left(x_{1}, 1,0, \ldots, 0 ; A\right)=H_{n_{1}, \ldots, n_{p}}\left(x_{1} ; A\right)
$$

and

$$
H_{n_{1}, \ldots, n_{p}}\left(-x_{1}, \ldots, x_{p} ; A\right)=(-1)^{n_{1}+\cdots+n_{p}} H_{n_{1}, \ldots, n_{p}}\left(x_{1}, \ldots, x_{p} ; A\right) .
$$

Some recurrence relations have been deduced for the p-index and p-variable Hermite matrix polynomials. We record the following theorem.

Theorem 4.4. The p-index $p$-variable Hermite matrix polynomials satisfies the following relations:

$$
\begin{aligned}
& \frac{\partial^{s}}{\partial x_{1}^{s}} H_{n_{1}, \ldots, n_{p}}\left(x_{1}, \ldots, x_{p} ; A\right) \\
& =(\sqrt{2 A})^{s} \sum_{r_{1}=0}^{s} \cdots \sum_{r_{p-1}=0}^{r_{p-2}} \frac{s ! n_{1} ! \cdots n_{p} ! H_{n_{1}-\left(s-r_{1}-\cdots-r_{p-1}\right), n_{2}-r_{1}, \ldots, n_{p}-\left(r_{p-1}-r_{p}\right)}\left(x_{1}, \ldots, x_{p} ; A\right)}{\left(n_{1}-\left(s-r_{1}-\cdots-r_{p-1}\right) !\left(n_{2}-r_{1}\right) ! \cdots\left(n_{p}-r_{p-1}\right) ! r_{1} ! \cdots r_{p-1} !\left(s-r_{1}-\cdots-r_{p-1}\right) !\right.}, \\
& \frac{\partial^{s}}{\partial x_{2}^{s}} H_{n_{1}, \ldots, n_{p}}\left(x_{1}, \ldots, x_{p} ; A\right) \\
& =(-1)^{s} \sum_{r_{1}=0}^{n_{2}} \cdots \sum_{r_{p-1}=0}^{n_{p}} \frac{(2 s) ! n_{1} ! \cdots n_{p} ! H_{n_{1}-\left(s-r_{1}-\cdots-r_{p-1}\right), n_{2}-r_{1}, \ldots, n_{p}-\left(r_{p-1}-r_{p}\right)}\left(x_{1}, \ldots, x_{p} ; A\right)}{\left(n_{1}-\left(s-r_{1}-\cdots-r_{p-1}\right) !\left(n_{2}-r_{1}\right) ! \cdots\left(n_{p}-r_{p-1}\right) ! r_{1} ! \cdots r_{p-1} !\left(s-r_{1}-\cdots-r_{p-1}\right) !\right.},
\end{aligned}
$$

Proof. By Differentiating (4.1) with respect to " $x_{1}$ ", we get

$$
\begin{aligned}
& \sqrt{(2 A)}\left(t_{1}+\cdots+t_{p}\right) \sum_{n_{1}, \ldots, n_{p}=0}^{\infty} H_{n_{1}, \ldots, n_{p}}\left(x_{1}, \ldots, x_{p} ; A\right) \frac{t_{1}^{n_{1}}}{n_{1} !} \cdots \frac{t_{p}^{n_{p}}}{n_{p} !} \\
& =\sum_{n_{1}, \ldots, n_{p}=0}^{\infty} \frac{\partial}{\partial x_{1}} H_{n_{1}, \ldots, n_{p}}\left(x_{1}, \ldots, x_{p} ; A\right) \frac{t_{1}^{n_{1}}}{n_{1} !} \cdots \frac{t_{p}^{n_{p}}}{n_{p} !}
\end{aligned}
$$

which on employing the result

$$
\begin{aligned}
\left(z_{1}+\cdots+z_{p}\right)^{n}= & \sum_{r_{1}=0}^{n} \sum_{r_{2}=0}^{n-r_{1}} \cdots \sum_{r_{p-1}=0}^{n-r_{1}-\cdots-r_{p-2}}\left(\begin{array}{c}
n \\
r_{1}
\end{array}\right)\left(\begin{array}{c}
n-r_{1} \\
r_{2}
\end{array}\right) \cdots\left(\begin{array}{c}
n-r_{1}-\cdots-r_{p-2} \\
r_{p-1}
\end{array}\right) \\
& z_{1}^{n-r_{1}-\cdots-r_{p-1}} z_{2}^{r_{1}} z_{3}^{r_{2}} \cdots z_{p}^{r_{p-1}},
\end{aligned}
$$

and on comparing the coefficients of $t_{1}^{n_{1}} t_{2}^{n_{2}} \cdots t_{p}^{n_{p}}$ and then on iteration we get (4.3). Similarly, (4.4) can be established.

We conclude this section giving Rodrigues formula for the p-index and p-variable Hermite matrix polynomials. 
Theorem 4.5. Let $A$ be matrix in $C^{N \times N}$ such that $\Re(\lambda)>0$ for every eigenvalue $\lambda \in \sigma(A)$. Then the $p$-index and p-variable Hermite matrix polynomials has the following representation

$$
H_{n_{1}, \ldots, n_{p}}\left(x_{1}, \ldots, x_{p} ; A\right)=\exp \left[-\left(x_{2}+\cdots x_{p}\right)(2 A)^{-1} \frac{\partial^{2}}{\partial x_{1}^{2}}\right]\left(x_{1} \sqrt{(2 A)}\right)^{n_{1}+\cdots+n_{p}}
$$

Proof. We refer to the proof of (3.1).

\section{The p-index and p-variable Chebyshev and Legendre matrix polynomials}

The p-index and p-variable Hermite matrix polynomials will be exploited here to define a matrix version of Chebyshev and Legendre polynomials. We recall that the Chebyshev and Legendre polynomials of the second kind [2] are defined by:

$$
U_{n}(z)=\sum_{k=0}^{\left[\frac{n}{2}\right]} \frac{(-1)^{k}(n-k) !(2 z)^{n-2 k}}{k !(n-2 k) !}
$$

and

$$
P_{n}(x)=\sum_{k=0}^{\left[\frac{n}{2}\right]} \frac{(-1)^{k}\left(\frac{1}{2}\right)_{n-k}(2 x)^{n-2 k}}{k !(n-2 k) !}=\sum_{k=0}^{\left[\frac{n}{2}\right]} \frac{\left.(-1)^{k}(2 n-2 k)\right) !(2 x)^{n-2 k}}{2^{2 n-2 k} k !(n-k) !(n-2 k) !},
$$

respectively. Suppose that $A$ is a matrix in $C^{N \times N}$ such that $\operatorname{Re}(\lambda)>0$,for every eigenvalue $\lambda \in \sigma(A)$, where $\sigma(A)$ denotes the set of all the eigenvalues of $A$. Then, we define the p-index and p-variable Chebyshev and Legendre matrix polynomials by the integral representation:

$$
U_{n_{1}, \ldots, n_{p}}\left(x_{1}, \ldots, x_{p} ; A\right)=\frac{1}{n_{1} ! \cdots n_{p} !} \int_{0}^{\infty} e^{-t} t^{n_{1}+\cdots+n_{p}} H_{n_{1}, \ldots, n_{p}}\left(x_{1}, \frac{x_{2}}{t}, \ldots, \frac{x_{p}}{t} ; A\right) d t,
$$

and

$$
P_{n_{1}, \ldots, n_{p}}\left(x_{1}, \ldots, x_{p} ; A\right)=\frac{2}{n_{1} ! \cdots n_{p} ! \sqrt{\pi}} \int_{0}^{\infty} e^{-2 t} t^{n_{1}+\cdots+n_{p}} H_{n_{1}, \ldots, n_{p}}\left(x_{1} t, x_{2}, \ldots, x_{p} ; A\right) d t,(5
$$

respectively.

Starting from (5.1) and applying the result

$$
\int_{0}^{\infty} e^{-t} t^{n} d t=n !
$$

we can conclude that

$$
\begin{aligned}
\frac{1}{n_{1} ! \cdots n_{p} !} \int_{0}^{\infty} t^{n_{1}+\cdots+n_{p}} e^{-t} H_{n_{1}, \ldots, n_{p}}\left(\frac{x_{1}}{t}, \ldots, \frac{x_{p}}{t} ; A\right) d t \\
=\sum_{r_{1}=0}^{\left[\frac{n_{1}}{2}\right]} \cdots \sum_{r_{p}=0}^{\left[\frac{n_{p}}{2}\right]} \frac{(-1)^{r_{1}+\cdots+r_{p}}\left(2 r_{1}+\cdots+2 r_{p}\right) !\left(n_{1}+\cdots+n_{p}-\left(r_{1}+\cdots+r_{p}\right)\right) !}{\left(r_{1}+\cdots+r_{p}\right) !\left(n_{1}-2 r_{1}\right) ! \cdots\left(n_{p}-2 r_{p}\right) !} \\
\frac{\left(x_{1} \sqrt{2 A}\right)^{n_{1}+\cdots+n_{p}-2 r_{1}-\cdots-2 r_{p}}\left(x_{2}+\cdots+x_{p}\right)^{r_{1}+\cdots+r_{p}}}{\left(2 r_{1}\right) ! \cdots\left(2 r_{p}\right) !} .
\end{aligned}
$$


Thus, we find that

$$
\begin{aligned}
U_{n_{1}, \ldots, n_{p}}\left(x_{1}, \ldots, x_{p} ; A\right) \\
=\sum_{r_{1}=0}^{\left[\frac{n_{1}}{2}\right]} \cdots \sum_{r_{p}=0}^{\left[\frac{n_{p}}{2}\right]} \frac{(-1)^{r_{1}+\cdots+r_{p}}\left(2 r_{1}+\cdots+2 r_{p}\right) !\left(n_{1}+\cdots+n_{p}-\left(r_{1}+\cdots+r_{p}\right)\right) !}{\left(r_{1}+\cdots+r_{p}\right) !\left(n_{1}-2 r_{1}\right) ! \cdots\left(n_{p}-2 r_{p}\right) !} \\
\\
\frac{\left(x_{1} \sqrt{2 A}\right)^{n_{1}+\cdots+n_{p}-2 r_{1}-\cdots-2 r_{p}}\left(x_{2}+\cdots+x_{p}\right)^{r_{1}+\cdots+r_{p}}}{\left(2 r_{1}\right) ! \cdots\left(2 r_{p}\right) !} .
\end{aligned}
$$

Similarly, proceeding on the same lines and instead of integral formula (5.3) using the integral formula

$$
\int_{0}^{\infty} e^{-t^{2}} t^{n} d t=\frac{1}{2} \Gamma\left(\frac{1}{2} n+\frac{1}{2}\right)
$$

and applying Legendre duplication formula [8,p17(14)]:

$$
\Gamma\left(n+\frac{1}{2}\right)=\frac{\sqrt{\pi}(2 n) !}{2^{2 n} n !}, \quad \text { or } \quad\left(\frac{1}{2}\right)_{n}=\frac{(2 n) !}{2^{2 n} n !}, \quad(n=0,1,2, \ldots) .
$$

we obtain

$$
\begin{aligned}
& P_{n_{1}, \ldots, n_{p}}\left(x_{1}, \ldots, x_{p} ; A\right) \\
&=\sum_{r_{1}=0}^{\left[\frac{n_{1}}{2}\right]} \cdots \sum_{r_{p}=0}^{\left[\frac{n_{p}}{2}\right]} \frac{(-1)^{r_{1}+\cdots+r_{p}}\left(2 r_{1}+\cdots+2 r_{p}\right) !\left(\frac{1}{2}\right)_{n_{1}+\cdots+n_{p}-r_{1}-\cdots-r_{p}}}{\left(r_{1}+\cdots+r_{p}\right) !\left(n_{1}-2 r_{1}\right) ! \cdots\left(n_{p}-2 r_{p}\right) !} \\
& \frac{\left(x_{1} \sqrt{2 A}\right)^{n_{1}+\cdots+n_{p}-2 r_{1}-\cdots-2 r_{p}}\left(x_{2}+\cdots+x_{p}\right)^{r_{1}+\cdots+r_{p}}}{\left(2 r_{1}\right) ! \cdots\left(2 r_{p}\right) !}, \\
&= \sum_{r_{1}=0}^{\left[\frac{n_{1}}{2}\right]} \ldots \sum_{r_{p}=0}^{\left[\frac{n_{p}}{2}\right]} \frac{(-1)^{r_{1}+\cdots+r_{p}}\left(2 r_{1}+\cdots+2 r_{p}\right) !\left(2 n_{1}+\cdots+2 n_{p}-2 r_{1}-\cdots-2 r_{p}\right) !}{2^{2\left(n_{1}+\cdots+n_{p}-r_{1}-\cdots-r_{p}\right)}\left(r_{1}+\cdots+r_{p}\right) !\left(n_{1}-2 r_{1}\right) ! \cdots\left(n_{p}-2 r_{p}\right) !} \\
& \frac{\left(x_{1} \sqrt{2 A}\right)^{n_{1}+\cdots+n_{p}-2 r_{1}-\cdots-2 r_{p}}\left(x_{2}+\cdots+x_{p}\right)^{r_{1}+\cdots+r_{p}}}{\left(n_{1}+\cdots+n_{p}-r_{1}-\cdots-r_{p}\right) !\left(2 r_{1}\right) ! \cdots\left(2 r_{p}\right) !} .
\end{aligned}
$$

\section{Acknowledgement}

The first author M. A. Pathan would like to thank the Department of Science and Technology, Government of India, for the financial assistance for this work under project number SR/S4/MS:794/12and the Centre for Mathematical and Statistical Sciences for the facilities.

\section{References}

[1] R. S. Batahan, A new extension of Hermite matrix polynomial and its applications, LinearAlgebra Appl., 419 (2006), 82-92.

[2] P. J. Davis, Interpolation and Approximation, Vover, Now York, 1975. 
[3] L. Jodar, E. Defez and E. Ponsoda, Orthogonal matrix polynomials with respect to liner matrix moment functionals; Theory and Applications, J.Approx. Theory Applications, (12)1 (1996), 96-115.

[4] L. Jodar and R. Company, Hermite matrix polynomials and second order matrix differential equations, J. Approx. Theory Applications, (12)2 (1996), 20-30.

[5] L. Jodar, R. Company and E. Navarro, Laguerre matrix polynomials and system of second order differential equations, Appl. Numer. Math., 15 (1994), 53-63.

[6] K. A. M. Sayyed, M. S. Metwally and R. S. Batahan, Gegenbauer matrix polynomials and second order matrix differential equations, Div., Math., (12)2 (2004), 101-115.

[7] K.A. M. Sayyed, M. S. Metwally and R.S. Batahan, On generalized Hermite matrix polynomials, Electronic Journal of linear Algebra, 10 (2003), 272-279.

[8] H. M. Srivastava and H. L. Manocha, A Treatise on Generating Functions, Halsted press, John Wiley and Sons., New York, 1984.

Centre for Mathematical and statistical Sciences (CMSS), KFRI, Peechi P.O., Thrissur, Kerala-680653, India.

E-mail: mapathan@gmail.com

Department of Mathematics, Aden University, Kohrmakssar P.O.Box 6014, Yemen.

E-mail: mgbinsaad@Yahoo.com

Department of Mathematics, Aden University, Kohrmakssar P.O.Box 6014, Yemen.

E-mail: fadhl-Alsarhi@hotmail.com 